\title{
X-ray Reflectivity Study of Formation of Multilayer Porous Anodic Oxides of Silicon
}

\author{
R. Fenollosa ${ }^{1}, \mathrm{H} . \mathrm{You}^{2}$, and Y. Chu ${ }^{3}$, and V. Parkhutik ${ }^{1}$ \\ 'Dep. Termodinamica Aplicada, Univ. Politécnica de Valencia, Cami de Vera s/n. 46071 Valenica, Spain \\ ${ }^{2}$ Argonne National Laboratory, 9700 S. Cass Avenue, Argonne, IL 60439, USA
}

Materials Science Division
Argonne National Laboratory
Argonne, IL 60439
$\begin{aligned} & \text { The submitted manuscript has been created by } \\ & \text { the University of Chicago as Operator of Argonne } \\ & \text { National Laboratory ("Argonne") under Contract } \\ & \text { No. W-31-109-ENG-38 with the U.S. Department } \\ & \text { of Energy. The U.S. Government retains for } \\ & \text { itself, and others acting on its behalf, a paid-up, } \\ & \text { non exclusive, irrevocable worldwide license in } \\ & \text { said article to reproduce, prepare derivative } \\ & \text { works, distribute copies to the public, and } \\ & \text { perform publicly and display publicly, by or on } \\ & \text { behalf of the Government. }\end{aligned}$

July 1999

/jc

Distribution:

\section{1-2. PRS}

3. J. M. Gibson

4. P. A. Montano

5. F. Y. Fradin

6. Office of Science

7. Editorial Office

8. Authors

Paper submitted to European Materials Society Meeting, June 1 - 4, 1999, Strasbourg, France.

This work is supported by the Division of Materials Sciences, Office of Basic Energy Sciences of DOE, under contract No. W-31-109-ENG-38. Conducted as part of the project MAT-98-0342 financed by the Ministry of Culture and Education of Spain. 


\section{DISCLAIMER}

This report was prepared as an account of work sponsored by an agency of the United States Government. Neither the United States Government nor any agency thereof, nor any of their employees, make any warranty, express or implied, or assumes any legal liability or responsibility for the accuracy, completeness, or usefulness of any information, apparatus, product, or process disclosed, or represents that its use would not infringe privately owned rights. Reference herein to any specific commercial product, process, or service by trade name, trademark, manufacturer, or otherwise does not necessarily constitute or imply its endorsement, recommendation, or favoring by the United States Government or any agency thereof. The views and opinions of authors expressed herein do not necessarily state or reflect those of the United States Government or any agency thereof. 


\section{DISCLAIMER}

Portions of this document may be illegible in electronic image products. Images are produced from the best available original document. 


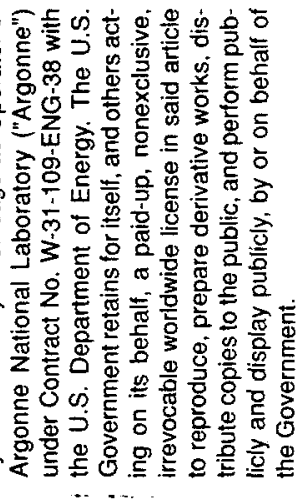

\title{
X-RAY REFLECTIVITY STUDY OF FORMATION OF MULTILAYER POROUS ANODIC OXIDES OF SILICON
}

\author{
R. Fenollosa ${ }^{* 1}$, H. You ${ }^{2}$ and Y. Chu' ${ }^{2}$ V. Parkhutik ${ }^{1}$ \\ ${ }^{1}$ Dep. Termodinámica Aplicada, Univ. Politécnica de Valencia, \\ Camí de Vera s/n, 46071 Valencia, Spain, \\ ${ }^{2}$ Argonne National Laboratory 9700 S. Cass Avenue, Argonne, IL \\ 60439 , USA.
}

\begin{abstract}
The paper reports data on the kinetics of anodic oxide films growth on silicon in aqueous solutions of phosphoric acids as well as a study of the morphology of the oxides grown in a special regime of the oscillating anodic potential. X-ray reflectivity measurements were performed on the samples of anodic oxides using an intense sinchrotron radiation source. They have a multilayer structure as revealed by theoretical fitting of the reflectivity data. The oscillations of the anodic potential are explained in terms of synchronized oxidation/dissolution reactions at the silicon surface and accumulation of mechanic stress in the oxide film.
\end{abstract}

\section{Keywords}

Electrochemical oscillations, anodic silicon oxide, x-ray reflectivity.

\section{Introduction}

The oscillations of the anodic potential during polarization of silicon wafers galvanostatically in strong inorganic acids is a very interesting phenomenon which origin still remains unclear despite intensive research [1-4]. The oscillation amplitude and period are the function of the electrolyte temperature and concentration as well as of anodic current density [1]. Chemical composition, structure, and physical properties of the oxides grown during the oscillatory phenomenon are not very well understood.

In this paper we report the study of the morphology of the anodic oxides at different stages of the oscillatory anodic kinetics using the method of reflectivity of $x$-rays obtained from the intense synchrotron radiation source to provide a deeper insight into the mechanism of the electrochemical oscillations.

\footnotetext{
* Corresponding Author: Roberto Fenollosa Tel: +34963877320, Fax: +34963877329 E-mail: rfenollo@ter.upv.es
} 


\section{Experimental set-up}

p-Si (100) wafers (0.01-6 Ohm.cm) were used in the work. The choice of the conductivity type and doping level was not critical in the present case as the reactive layer is not the silicon but rather its anodic oxide. Samples of $1 \times 1 \mathrm{~cm}^{2}$ were anodized galvanostatically at different current densities and temperatures of the electrolyte in a conventional three-electrode electrolytic cell in $1 \mathrm{M}$ phosphoric acid. The temperature of the electrolyte was controlled with a precision of $\pm 1^{\circ} \mathrm{C}$.

$\mathrm{X}$-ray reflectivity measurements were made at the X10A beamline of the Brookhaven National Laboratory Synchrotron. Focused monochromatic beam of x-rays (wavelength $1.499 \AA$ ) was used. Reflectivity spectra were obtained by monitoring the ratio of reflected to incident intensity as a function of the perpendicular component of the wavevector, $q_{z}$. Variation in $q_{z}$ was achieved by changing the angle of incidence and reflection.

\section{Experimental Results and discussion}

\subsection{Kinetics of the oxide growth}

Fig.1.a. illustrates kinetics of the anodic oxide growth with polarizing the samples galvanostatically at the same current density $\left(1.6 \mathrm{~mA} \cdot \mathrm{cm}^{-2}\right)$ at different temperatures of electrolyte. The oscillations are better shaped when the temperature is increasing and the current density is decreasing. The kinetics looses the oscillatory feature at low temperatures. Fig.1.b. shows kinetic curves corresponding to samples anodized at different current densities and the same temperature of electrolyte $\left(26^{\circ} \mathrm{C}\right)$. The oscillations are enhanced by lower current densities.



Fig.1. Kinetics of p-Si anodization in $1 \mathrm{M} \mathrm{H}_{3} \mathrm{PO}_{4}$. at: (a) current density of $1.6 \mathrm{~mA} \cdot \mathrm{cm}^{-2}$ and different temperatures of the electrolyte; (b) constant temperature of electrolyte of $26^{\circ} \mathrm{C}$ and for different current densities. The curves are shifted for $10 \mathrm{~V}$ each to avoid overlapping 


\subsection{X-ray reflectivity data and their fit}

In characteristic points of the oscillatory kinetics of the oxide growth (basically corresponding to maximums and minimums of the potential) the anodization process was stopped. In such a way a series of the samples was formed representing the different moments of the oscillatory oxide growth. Upon switching off the voltage, each sample was removed from the electrolyte, washed in distilled water and dried.

Fig.2.a. shows the $x$-ray reflectivity curves from five samples. The first one $(0)$ corresponds to a non-etched sample and it shows a long period oscillation that indicates the presence of a very thin layer on the top of the silicon crystal. Curves (1.1 to 1.4) correspond to samples anodized at the same current density $\left(1 \mathrm{~mA} / \mathrm{cm}^{2}\right)$ and temperature $\left(55^{\circ} \mathrm{C}\right)$ until reaching different stages (specified in the inset by arrows) of the oscillatory kinetics.
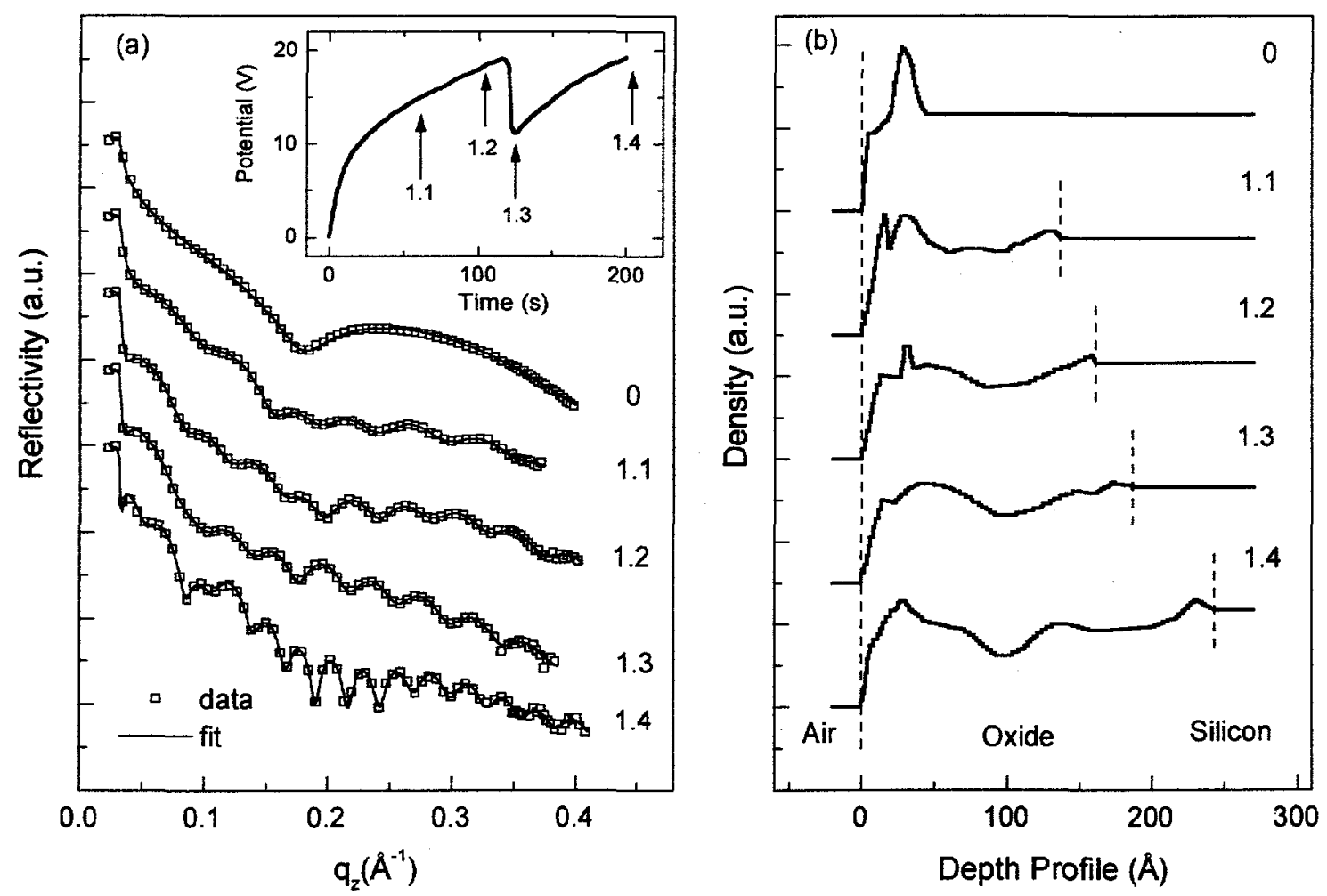

Fig.2. (a) Reflectivity scans from the non-treated (0) and anodized (1.1 - 1.4) samples. The inset shows its kinetic curve and the arrows mark the moments when the anodization process was stopped. The experimental points are rarefied three times to make fit curves visible.

(b) Corresponding density profiles.

In order to determine quantitatively the morphology features for the oxide films formed during the voltage oscillations, the reflectivity data were fitted using the Spreadsheet Environment Reflectivity Fitting (SERF) software developed by C. Co and K. A. Welp [5]. The input model includes a stack of flat layers whose thickness and refractive index are taken as adjustable parameters and embedded gradient layers of thickness as adjustable parameter and refractive index varying according to the error function between the refractive indexes of the adjacent flat layers. Taking into account that the refractive index of one medium is proportional to its density, the final input model can be expressed in terms of thickness and density. The SERF program calculates the reflectivity at each $q_{z}$ using a 
dynamics method introduced by Parratt [6] and the input model and fits to the reflectivity experimental data.

The depth profiles obtained from the fitting procedure are shown in Fig.2.b. Curve 0 corresponds to a dense layer (hereafter layer 0 ) of $45 \AA$ thick on the top of the silicon crystal. We attribute it to a surface oxide-like substance preexisting at the surface of silicon wafers. Curves 1.1 to 1.4 can be divided into three parts. The very external part corresponds to the partially etched layer 0 , the intermediate one responds for the anodic oxide and the internal one corresponds to the pure silicon crystal.

The layer corresponding to the anodic oxide, in its turn, also consists of two parts. The one adjacent to the silicon wafer (hereafter layer I ) possesses a density higher than that typical of the amorphous $\mathrm{SiO}_{2}\left(\rho_{\mathrm{a}-\mathrm{SiO} 2}=1.9 \mathrm{~g} / \mathrm{cm}^{3}\right)$ reaching a maximum value of $2.5 \mathrm{~g} / \mathrm{cm}^{3}$, and its thickness is about $25 \AA$. This layer appears nearly unchanged in all the curves and its elevated density value may be related with the volume built-up during the oxide formation at $\mathrm{Si}_{-} \mathrm{SiO}_{2}$ interface. The depth profile shape of the layer that extends from the upper part of layer I to the bottom of layer 0 (hereafter layer II), varies periodically according to the potential oscillation.

The stage 1.1 yields a layer II of about $60 \AA$ and consists of a series of sub-layers whose density is lower than that of $\rho_{\mathrm{a}-\mathrm{SiO} 2}$. We attribute this decrease in density to the formation of the pores. The value of porosity $P$ can be estimated by using Eqn.1., where $L_{i}$ is the thickness and $\rho_{i}$ the density of sub-layer $i$ and the sums extend to all the sub-layers of the zone.

$$
P=\left(1-\frac{\sum_{i} L_{i} \times \rho_{i}}{\rho_{a-S_{i} O_{2}} \times \sum_{i} L_{i}}\right) \times 100
$$

The result is $P=6 \%$ at stage 1.1. When the oscillation is in its first maximum (stage 1.2), zone II spreads to get a shape of a valley, its thickness increases to $80 \AA$ and $P$ reaches $12 \%$. After the first drop of the anodic voltage (stage 1.3) density distribution experiences an essential change. The second density minimum appears on the top of layer I and the overall oxide thickness increases for $20 \AA$ more. Meanwhile the thickness of the first valley-like minimum remains constant and its porosity increases to $18 \%$. At the stage $1.4\left(2^{\text {nd }}\right.$ maximum of the anodic voltage), the first minimum does not experience any essential change and the second one consolidates. Its thickness and porosity reach the same values than those of the first minimum at the stage 1.2. This behavior repeats periodically as the anodic oscillation progresses as it can be observed in Fig.3, where we have plotted the reflectivity curves and depth profiles for samples anodized at the same current density and temperature than those of Fig.2. and removed from the electrolyte at stages $2.1,2.2$. and 2.3 , corresponding to the $2^{\text {nd }}$ maximum, $2^{\text {nd }}$ minimum and $3^{\text {rd }}$ maximum of the potential.

\subsection{The model for the multilayer oxide growth}

It is possible to distinguish two regimes in each kinetic curve. During the first one $(0 \mathrm{~s}<\mathrm{t}<10 \mathrm{~s}$ approximately), a very dense silicon oxide passive layer $\left(\rho>\rho_{\mathrm{a}-S i O 2}\right)$ grows. Its thickness is about $25 \AA$ (layer I). This stage corresponds to gradual increase of the anodic potential. During the second stage $(t>10 \mathrm{~s})$, there are two processes running at the same time: the growth of layer I plus its local dissolution on the top side. The reason why the dissolution process appears is explained earlier [7] applying the assumption of the local field-enhanced oxide dissolution. This model assumes that the oxide growth and dissolution are both non-linearly dependent on the electric field strength which, in its turn, is two-dimensionally distributed in the vicinity of the pore tips. 
With increasing the etching time, the pores can change their shapes (from needle like to bottle-like) depending on electrochemical parameters (current density, temperature and concentration of electrolyte). The competition of oxidation and dissolution processes increases the thickness and porosity of the layer II while the layer I remains intact.

Appearance of the pores in the region II and their widening (as the density of the layer decreases with time) facilitate the penetration of the electrolyte inside the oxide thus diminishing the potential necessary to maintain the constancy of the current density. The thickness increase has the opposite effect. The balance between both processes is shifted towards the increase in porosity, producing a decrease in the kinetic curve slope.
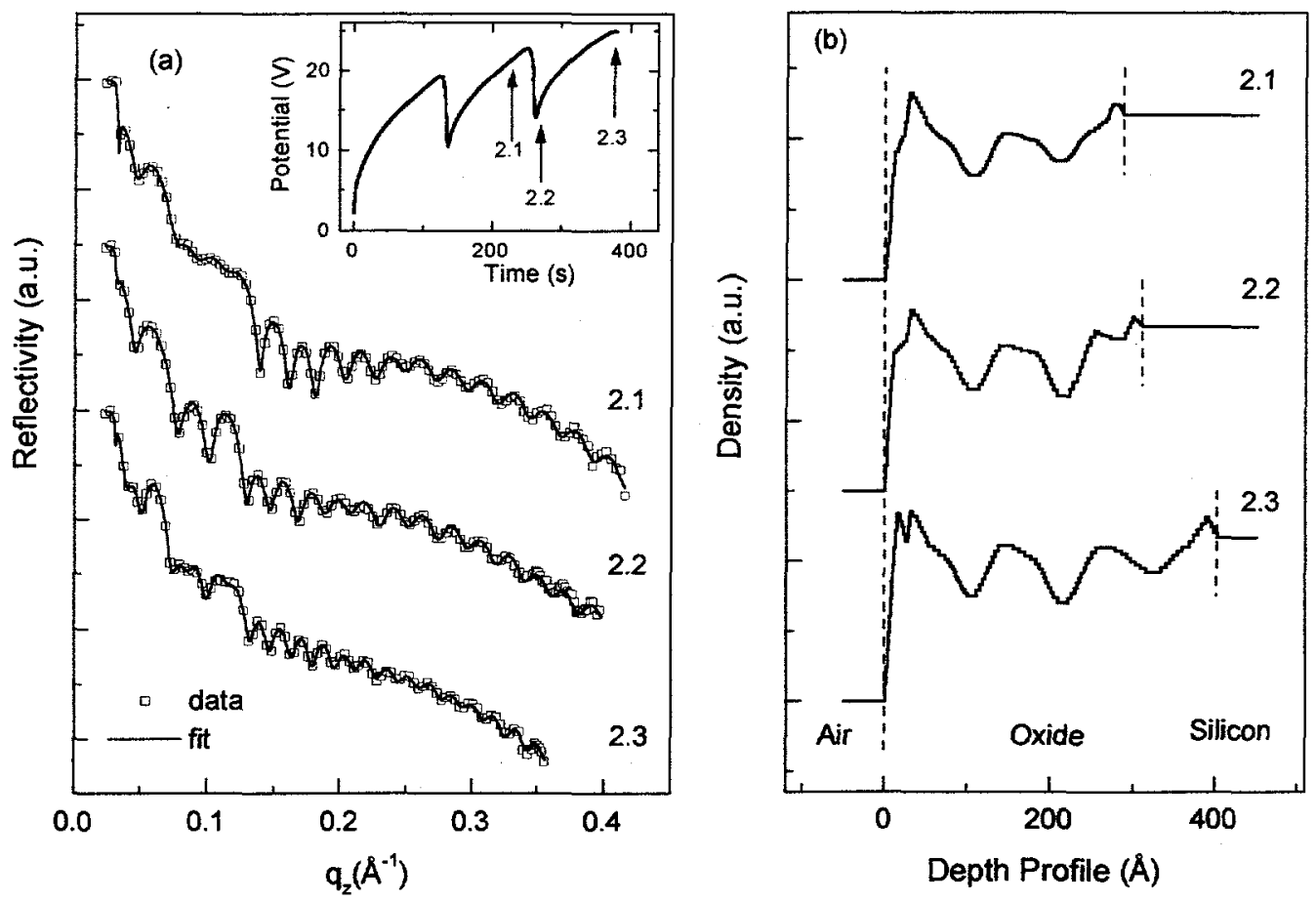

Fig.3. (a) Reflectivity scans from the anodized samples $2.1-2.3$. The inset shows kinetics of the oxide growth and the arrows mark the moments when its anodization process was stopped. (b) Corresponding density profiles.

The oscillating anodic process results in accumulation and release of stress inside the oxide layer. This stress has been first studied by Lehmann [3]. Our data reveal the existence of very dense thin oxide layer at the interface $\mathrm{Si}-\mathrm{SiO}_{2}$ which is probably the result of the volume built-up when the oxide is forming (the Piiling-Bedworth ratio for silicon is higher than 1). The accumulation of the stress is assumed to be one of the important factors in the formation of the porous layers [8]. We believe that the stress can further enhance the local dissolution of silicon dioxide through generation of the defects which provide easy paths for dissolution process. The sharp increase of the porosity (from $12 \%$ to $18 \%$ in our estimations) in the moment when the anodic potential reaches its minimum may be related with this effect. This point needs further study.

\section{Conclusions.}


$\mathrm{X}$-ray reflectivity studies performed on the oxide layers formed on silicon in a regime of oscillating anodic potential show unambiguously that the oxides possess the multilayer structure. Each sublayer is separated from others by a layer of less-dense substance, presumably oxide with high porosity. The number of layers is equal to the number of the oscillations experienced by the sample during its polarization.

\section{Acknowledgements}

The work was conducted as a part of the project MAT-98-0342 financed by the Ministry of Culture and Education of Spain. The authors wish to acknowledge C. Co and K.A. Welp for permission to download and use their software. One of us (R. F.) is indebted to the Ministry of Education and Culture of Spain for providing him a grant to work at Argonne and Brookhaven National Laboratories.

\section{References}

[1] V. Parkhutik, Electrochimica Acta. 36 (1991) 1611.

[2] O. Nast, S. Rauscher, H. Jungblut, H. J. Lewerenz, J. Electroan. Chem. 422 (1998) 169

[3]V. Lehmann, J. Electrochem. Soc. 143 (1996) 1313

[4] F. Ozanam, J.N. Chazalviel, A. Radi and M. Etman, J. Electrochem. Soc. 139 (1992) 2491

[5] C. Co and K. A. Welp, SERF., Univ. Delaware, Dep. Chem. Eng. Newark, DE 19716 USA

[6] F. Parratt, Phys. Rev. 92 (1954) 359.

[7] Yu. Makushok, V. Parkhutik, J.M. Martinez-Duart and J.M. Albella, J. Phys. 27 (1994) 661.

[8] V.Parkhutik, Mate.Sci.and Engineer., B58(1999)95. 